\title{
Éditorial
}

\section{Gingivites ou gingivopathies : à vous de choisir !}

\author{
Jean-Christophe Fricain
}

Il est des classifications qui perdurent. À l'heure de « l’Evidence Based Dentistry », on pourrait s'attendre à un chamboulement ou tout au moins à une évolution des concepts. Malheureusement, le manque de moyens, la dispersion, la jeunesse universitaire de l'odontologie, et je ne sais quoi encore, empêchent la remise en cause des dogmes. Le cloisonnement entre spécialités médicales, et encore plus avec l'odontologie, est un obstacle à une évolution commune des idées et des habitudes. La médecine buccale en est la parfaite illustration. L'otorhinolaryngologiste explore l'oropharynx, l'odontologiste les dents et la gencive, le dermatologue les lèvres et la langue, le médecin généraliste la langue et l'oropharynx. Le stomatologiste explorait l'ensemble de la muqueuse ; sa formation généralement réduite en parodontologie minimisait souvent la pertinence des diagnostics et des traitements proposés. L'arrivée des chirurgiens oraux comblera certainement les lacunes inhérentes aux formations des spécialités précitées.

En attendant, force est de constater que la dualité odontologie-médecine est forte et qu'elle n'est pas sans conséquences sur la conception de la pratique. Pour illustrer mon propos, je voudrais revenir sur la classification des gingivites. La dernière classification qui fait référence a été proposée en 1999 suite à un workshop international, puis synthétisée et publiée par GC Armitage [1]. Cette classification est basée sur l'étiologie et sépare les gingivites dues au biofilm des gingivites sans lien avec la plaque. Le premier risque d'une telle séparation est de porter systématiquement le diagnostic de gingivite due à la plaque lorsque celle-ci est présente. Statistiquement, ce raccourci est juste dans la plupart des cas car $97 \%$ des gingivites sont dues au biofilm. Par contre, dès lors que la gingivite n'est pas due au biofilm, un retard diagnostique est observé. Il était de 19 mois pour les gingivites desquamatives dans l'étude française publiée par Vaillant [2]. Le second risque est de formater l'esprit des praticiens et de les focaliser sur la plaque dentaire plutôt que sur le tissu gingival. En d'autres mots, mettre la « charrue avant les bœufs » ou, en termes plus médicaux, rechercher une cause avant d'avoir posé un diagnostic. Le troisième risque qui découle des deux premiers est de réduire les maladies gingivales aux gingivites. En effet le terme gingivopathies serait plus adapté. Il recouvre l'ensemble des pathologies gingivales sans les réduire aux seules gingivites. La classification clinique des gingivopathies pourrait alors être basée sur la lésion élémentaire. Cette approche a été en partie privilégiée dans l'ouvrage de Dridi et al. [3]. L'adoption d'une telle classification aurait pour avantage de se rapprocher de la démarche diagnostique utilisée depuis longtemps par nos confrères dermatologues et plus récemment par les spécialistes de médecine buccale. La lésion élémentaire est la modification du tissu observée à l'examen clinique. Cette lésion élémentaire concerne aussi bien la gencive que le reste de la muqueuse buccale. Une fois identifiée la lésion élémentaire, le praticien se posera la question de l'étiologie puis du diagnostic de la maladie. Une telle approche permettrait de corriger les erreurs, le caractère peu pratique, les confusions et les manques de la classification dite d'Armitage [1]. À titre d'exemple, dans la partie consacrée aux gingivites dues à la plaque, la gingivite ulcéro nécrotique (GUN) est individualisée alors que les gingivites érythémateuses et hypertrophiques dues à la plaque ne le sont pas, et que sont listées toutes les gingivites associées à des facteurs favorisants locaux ou généraux. Ce mélange de lésions élémentaires et d'étiologies traduit le caractère confus de la classification. L'allergie est placée sur le même plan que les gingivites traumatiques ou associées aux dermatoses bulleuses, alors que les réactions d'hypersensibilité sont en pratique exceptionnelles. Parmi les erreurs, on peut citer la GUN qui n'apparaît que dans les gingivites dues à la plaque alors

\footnotetext{
* Correspondance : fricainj@aol.com
} 
qu'elle peut s'observer dans une dermatose bulleuse ou dans une hémopathie maligne. Une autre erreur concerne les gingivites des leucémies : elles sont classées dans les gingivites dues à la plaque alors qu'elles peuvent s'observer indépendamment de la présence de biofilm, et être consécutives à l'infiltration des blastes. Enfin, le manque le plus critique de cette classification est l'absence de citation du carcinome épidermoïde gingival qui induit systématiquement une inflammation gingivale et par conséquent une gingivite...

Alors, gingivites ou gingivopathies ? À vous de choisir !

\section{Références}

1. Armitage GC. Development of a classification system for periodontal diseases and conditions. Ann Periodontol 1999;4:1-6.

2. Vaillant L, Chauchaix-Barthes $S$, Hüttenberger B, Machet $M$, Jan V, Goga D, Lorette G. Chronic desquamative gingivitis syndrome: retrospective analysis of 33 cases. Ann Dermatol Venerol 2000;127:381-7.

3. Dridi SM, Eijel AL, Gaultier F, Meyer J. La gencive pathologique de l'enfant à l'adulte. Information dentaire 2013;173-221. 